\title{
Roles of thermostable direct hemolysin (TDH) and TDH-related hemolysin (TRH) in Vibrio parahaemolyticus
}

\section{Pendru Raghunath* \\ Department of Microbiology, Dr. VRK Women's Medical College Teaching Hospital and Research Centre, Hyderabad, India}

\section{Edited by:}

Dongsheng Zhou, Beijing Institute of Microbiology and Epidemiology, China

\section{Reviewed by:}

Lingling Zhang, University of Texas Southwestern Medical Center at Dallas, USA

Learn-Han Lee, Monash University

Malaysia, Malaysia

\section{*Correspondence:}

Pendru Raghunath, Department of Microbiology, Dr. VRK Women's

Medical College Teaching Hospital and Research Centre,

R.R. District-500075, Hyderabad,

India

e-mail: raghunath_kmc@yahoo.co.in raghunathreddyp@gmail.com

\begin{abstract}
Vibrio parahaemolyticus is the leading cause of seafood borne bacterial gastroenteritis in the world, often associated with the consumption of raw or undercooked seafood. However, not all strains of $V$. parahaemolyticus are pathogenic. The thermostable direct hemolysin (TDH) or TDH-related hemolysin (TRH) encoded by $t d h$ and trh genes, respectively, are considered major virulence factors in $V$. parahaemolyticus. However, about $10 \%$ of clinical strains do not contain tdh and/or trh. Environmental isolates of $V$. parahaemolyticus lacking tdh and/or trh are also highly cytotoxic to human gastrointestinal cells. Even in the absence of these hemolysins, $V$. parahaemolyticus remains pathogenic indicating other virulence factors exist. This mini review aims at discussing the possible roles of $t d h$ and trh genes in clinical and environmental isolates of $V$. parahaemolyticus.
\end{abstract}

\section{Keywords: $V$. parahaemolyticus, $t d h$, trh, virulence factors}

\section{INTRODUCTION}

Vibrio parahaemolyticus is a Gram-negative, halophilic bacterium that occurs in estuarine environments worldwide ( $\mathrm{Su}$ and Liu, 2007; Nelapati et al., 2012; Ceccarelli et al., 2013; Zhang and Orth, 2013). It is the leading cause of seafood borne bacterial gastroenteritis in the world, often associated with the consumption of raw or undercooked seafood. However, not all strains of $V$. parahaemolyticus are pathogenic. Although mechanism by which $V$. parahaemolyticus causes enteric disease is not fully understood, clinical isolates most often produce either the thermostable direct hemolysin (TDH) or TDH-related hemolysin (TRH) encoded by $t d h$ and $t$ th genes, respectively (Zhang and Austin, 2005). TDH and TRH are considered major virulence factors in V.parahaemolyticus (Ceccarelli et al., 2013).

\section{CORRELATION OF $t d h$ AND trh WITH THE VIRULENCE OF V. parahaemolyticus}

Thermostable direct hemolysin exerts a variety of biological activities such as hemolytic activity, cytotoxicity, cardiotoxicity, and enterotoxicity. TDH is a pore-forming toxin, forms pores of $\sim 2 \mathrm{~nm}$ in diameter on erythrocyte membrane (Matsuda et al., 2010). The fairly large pore size allows both water and ions to flow through the membrane (Honda et al., 1992). These alterations in ion flux in the intestine is responsible for the diarrhea observed during infection. TRH is a heat labile toxin and immunologically similar to TDH (Honda et al., 1988). Both genes, $t r h$ and $t d h$ share approximately 70\% homology (Kishishita et al., 1992). Similar to TDH, TRH also activates $\mathrm{cl}^{-}$channels resulting in altered ion flux (Takahashi et al., 2000). Although TDH and TRH correlate with pathogenic strains, they do not fully account for $V$. parahaemolyticus pathogenicity (Lynch et al., 2005). Several studies have reported that some of the clinical strains do not contain tdh and/or trh (Jones et al., 2012; Li et al., 2014; Pazhani et al., 2014). Even in the absence of these hemolysins, V. parahaemolyticus remains pathogenic indicating other virulence factors exist (Jones etal., 2012). Mahoney etal. (2010) reported that environmental isolates of $V$. parahaemolyticus lacking $t d h$ and/or $t r h$ produced putative virulence factors like extracellular proteases, biofilm, siderophore, and highly cytotoxic to human gastrointestinal cells. Park et al. (2004a) reported that deletion of both copies of $t d h$ did not affect the cytotoxicity to HeLa cells and enterotoxicity assayed by the rabbit ileal loop test was lowered by $t d h$ deletion, but the mutant still showed partial fluid accumulation in rabbit intestine. Ming et al. (1994) reported that trh deletion resulted in partial but apparent fluid accumulation in ligated rabbit small intestine. These results clearly indicate that cytotoxicity and enterotoxicity of pathogenic $V$. parahaemolyticus are not explained by TDH and TRH alone and suggest that an unkown virulence factor (s) could be responsible for pathogenicity.

\section{TYPE III SECRETION SYSTEMS}

The type III secretion system (T3SS) of V. parahaemolyticus has been suggested as an important virulence factor is (Shimohata and Takahashi, 2010; Broberg et al., 2011). Two non-redundant T3SSs are reported from many V. parahaemolyticus strains (Park et al., 2004b; Broberg et al., 2011). Many studies suggest that T3SS1 is responsible for cytotoxicity, mouse lethality, and possibly induction of autophagy (Park et al., 2004b; Burdette et al., 2009; Hiyoshi et al., 2010). T3SS2 appears to be responsible for enterotoxicity and may play a role in the environmental fitness of strains (Park et al., 2004b; Hiyoshi et al., 2010; Matz et al., 2011). All V.parahaemolyticus isolates possess T3SS1 (Park et al., 2004b; Noriea et al., 2010). While T3SS2 is commonly associated with $V$. parahaemolyticus 
carrying $t d h$ and/or $t r h$. Two distinct lineages of T3SS2 have been described, showing correlations of $t d h$ with T3SS2 $\alpha$ and trh with T3SS2 $\beta$ (Park et al., 2004b; Noriea et al., 2010). However, recently T3SS2 $\beta$ has been detected in $t d h$ - and $t r h$-negative environmental strains of V. parahaemolyticus (Paranjpye et al., 2012). In another study, authors screened 77 clinical isolates of $V$. parahaemolyticus, which were submitted to the Centers for Disease Control and Prevention (CDC) in 2007 from wound infections or food-borne illness and reported that 21 of 77 (27\%) clinical V. parahaemolyticus strains were negative for $t d h$, trh, and T3SS2 (Jones et al., 2012). The results of these studies raise some concerns about the reliability of the $t d h$, trh, and T3SS2 genes as predictors of overall strain virulence.

\section{TYPE VI SECRETION SYSTEMS}

Comparison between pandemic and non-pandemic strains of $V$. parahaemolyticus led to identification of type VI secretion systems, T6SS1 (VP1386-VP1420) and T6SS2 (VPA1030-VPA1043), located on chromosome 1 and 2 of $V$. parahaemolyticus RIMD 2210633, respectively, (Boyd et al., 2008; Izutsu et al., 2008). The role of T6SS2 is under investigation, preliminary data suggested that the T6SS2 is not involved in cytotoxicity, helps in adhesion to host cells (Yu et al., 2012). Since T6SS2 and T3SS2 systems co-exist, it is proposed that both systems might cooperate during an infection process in host. T6SS2 initiates the first step of infection by adhering to host cells and T3SS2 exports effector molecules by inducing enterocytotoxicity (Yu et al., 2012). Role of T6SS1 has not yet been demonstrated. Recently, researchers suggested the role of T6SSs in environmental fitness of $V$. parahaemolyticus. Salomon etal. (2013) reported that T6SS1 is most active under warm marine-like conditions, while T6SS2 is active under low salt conditions. T6SS was used as a virulence marker to differentiate V. parahaemolyticus strains. Chao et al. (2010) reported that most pandemic strains isolated in China had the complete set of T6SS genes, whereas the majority of non-pathogenic strains had a partial set of T6SS genes.

\section{POSSIBLE ROLES OF tdh AND trh IN CLINICAL AND ENVIRONMENTAL ISOLATES OF V. parahaemolyticus}

First outbreak of foodborne gastroenteritis due to $V$. parahaemolyticus was reported in the year 1951 in Osaka, where people frequently consume raw or uncooked seafood (Fujino etal., 1953). Since then $V$. parahaemolyticus has reported from many food poisoning cases in Japan (Su and Liu, 2007; HaraKudo etal., 2012), in Taiwan (Yu etal., 2013), in China (Li et al., 2014), Bangladesh (Bhuiyan etal., 2002), HongKong, and Indonesia (Matsumoto etal., 2000). In India, a recent study, reported that $178 \mathrm{~V}$. parahaemolyticus strains were isolated from 13,607 diarrheal patients admitted in Infectious Diseases Hospital, Kolkata since 2001-2012 (Pazhani etal., 2014). Kanungo etal. (2012) have reported V. parahaemolyticus diarrheal cases from the urban slums of Kolkata, India.

The majority of clinical cases of V. parahaemolyticus have been associated with $V$. parahaemolyticus strains carrying $t d h$ and/or trh (Kanungo et al., 2012; Li et al., 2014; Pazhani et al., 2014). However, pathogenic strains including the pandemic clone have been rarely isolated from seafood and other environmental samples. This could be due to the occurrence of pathogenic strains in the estuarine environment at a lower level compared to non-pathogenic strains or that the pathogenic stains are more sensitive to dystrophic conditions in the aquatic environment and rapidly become non-culturable (Pace and Chai, 1989; Alam et al., 2002). We thought that host factors like bile or its component bile acids might trigger release from dormancy and increase virulence in V. parahaemolyticus strains. This could be the reason for selection of pathogenic strains in human gastrointestinal system inspite of low prevalence in aquatic environment. With this in view, a new enrichment broth containing bile salt, sodium taurocholate (ST broth) has been formulated and the efficiency of new enrichment broth in detecting and recovering pathogenic V. parahaemolyticus from seafood was compared with the traditional APW. Results of the study suggested that the ST broth is superior to APW for detection and isolation of pathogenic $V$. parahaemolyticus from seafood.

Previous studies suggested that expression of $t d h$ gene is upregulated under conditions simulating those in the human intestine (Gotoh etal., 2010; Broberg etal., 2011). Gotoh etal. (2010) are reported that TDH and T3SS2 proteins were detected in much higher concentrations when bacteria were cultured at 37 and $42^{\circ} \mathrm{C}$, which corresponds to the temperature of the intestine, than at lower temperatures. In the same study, they also identified bile as a potent stimulator of the production of TDH and T3SS2 proteins (Gotoh et al., 2010). Pathogenic strains may produce TDH and/or TRH more abundantly in hostile environment and these toxins might be helpful to acquire nutrients from host cells through their cytotoxic activities. This could be the reason for selection of pathogenic strains in human intestine compared to non-pathogenic strains. Within the human host, whether TDH and TRH play any other roles other than cytotoxicity and enterotoxicity of $V$. parahaemolyticus need to be studied. Bacterial pathogens frequently use environmental cues to discriminate between host and non-host environments. In response to these environmental cues, bacteria regulate their virulence gene expression for more efficient utilization of bacterial resources and facilitate colonization, leading to infection. V. parahaemolyticus, upon reaching a human host from environment, should expose to number of environmental cues such as temperature, $\mathrm{pH}$, osmolarity, oxygen levels, carbon sources, and concentration of various ions and compounds. In response to these environmental cues, $V$. parahaemolyticus carrying $t d h$ and/or trh might tightly coordinate their virulence associated genes expression. Whereas, $V$. parahaemolyticus strains lacking $t d h$ and/or trh may not be able to regulate their virulence associated genes expression and not able to establish colonization and infection in the human host. Mahoney etal. (2010) reported that in clinical strains carrying $t d h$ and/or trh, the expression of virulence associated traits including hemolysin, protease, motility, biofilm formation, and cytotoxicity correlated with increased temperature from 28 to $37^{\circ} \mathrm{C}$. In contrast, the environmental isolates did not induce their virulence associated traits in response to a temperature of $37^{\circ} \mathrm{C}$.

The occurence of $t d h$ and/or trh genes among environmental $V$. parahaemolyticus isolates is typically $1-10 \%$, but this depend 
on location, sample source and detection method. For example, we detected $t d h$ and $t r h$ genes in 20.7 and $41.4 \%$ of the seafood samples, respectively, from southwest coast of India by PCR after $18 \mathrm{~h}$ enrichment in ST broth. In the same study, we isolated $t d h$ and trh-carrying $V$. parahaemolyticus isolates from 19 to $44.8 \%$ of seafood samples, respectively, by colony hybridization following enrichment using ST broth (Raghunath et al., 2009). Kaysner et al. (1990) reported that between 49 and $78 \%$ of the sediment, water or oyster samples from Willapa Bay (WA, USA) contained trh-bearing V. parahaemolyticus. Alam et al. (2002) reported that $t d h$ and trh genes were positive in 55 and 20\% of environmental (water and sediment) samples, respectively, by MPN-PCR technique. But no $t d h$ and/or trh-carrying strains were isolated by the conventional MPN-culture procedure. The $t d h$ and $t r h$ genes are also present in non- $V$. parahaemolyticus vibrionaceae species such as V. mimicus, $V$. cholerae non-O1/non-O139, V. hollisae, V. diaboilcus, V. alginolyticus, and non-vibrio species such as Aeromonas veronii (Nishibuchi and Kaper, 1995; GonzalezEscalona et al., 2006; Raghunath et al., 2010; Shinoda, 2011; Klein et al., 2014). Such high frequency of these hemolysin genes in environmental strains of $V$. parahaemolyticus and occurance of these genes in the environmental strains of other Vibrio species indicate other potential roles of these hemolysins in the environment. Aquatic environment such as estuaries contain limited amount of nutrients. These hemolysins might be used to acquire nutrients through damage to cells of estuarine organisms. Matz et al. (2011) performed co-culture experiment of $V$. parahaemolyticus with a nanoflagellate Cafeteria roenbergensis and reported that the $t d h$ gene is required for the persistence of $V$. parahaemolyticus.

\section{CONCLUSION}

In conclusion, the reason for the selection of $V$. parahaemolyticus strains carrying $t d h$ and/or trh in the human host and the role of these hemolysins in coordinating virulence associated gene expression in response to the environmental cues to facilitate colonization and infection needs to addressed. The reason for the high frequency of $t d h$ and $t r h$ genes in environmental strains of $V$. parahaemolyticus is not clear. When compared to $t$ dh gene, the detection rate of trh gene in clinical strains is very less but relatively more in environmental strains. Pathogenic potential of these environmental strains of $V$. parahaemolyticus carrying trh gene needs to be studied. Future studies should also focus on the role of TDH and TRH in the environmental strains of $V$. parahaemolyticus for contribution of environmental fitness.

\section{REFERENCES}

Alam, M., Tomochika, K., Miyoshi, S., and Shinoda, S. (2002). Environmental investigation of potentially pathogenic Vibrio parahaemolyticus in the SetoInland Sea, Japan. FEMS Microbiol. Lett. 208, 83-87. doi: 10.1111/j.15746968.2002.tb11064.x

Bhuiyan, N. A., Ansaruzzaman, M., Kamruzzaman, M., Alam, K., Chowdhury, N. R., Nishibuchi, M., et al. (2002). Prevalence of the pandemic genotype of Vibrio parahaemolyticus in Dhaka, Bangladesh, and significance of its distribution across different serotypes. J. Clin. Microbiol. 40, 284-286. doi: 10.1128/JCM.40.1.284286.2002

Boyd, F. E., Cohen, L. V. A., Naughton, M. L., Ussery, W. D., Binnewies, T. T., Stine, C. O., et al. (2008). Molecular analysis of the emergence of pandemic Vibrio parahaemolyticus. BMC Microbiol. 8:110. doi: 10.1186/1471-2180$8-110$
Broberg, C. A., Calder, T. J., and Orth, K. (2011). Vibrio parahaemolyticus cell biology and pathogenicity determinants. Microbes Infect. 13, 992-1001. doi: 10.1016/j.micinf.2011.06.013

Burdette, D. L., Yarbrough, M. L., and Orth, K. (2009). Not without cause: Vibrio parahaemolyticus induces acute autophagy and cell death. Autophagy 5, 100-102. doi: 10.4161/auto.5.1.7264

Ceccarelli, D., Hasan, N. A., Hug, A., and Colwell, R. R. (2013). Distribution, and dynamics of epidemic, and pandemic Vibrio parahaemolyticus virulence factors. Front. Cell. Infect. Microbiol. 3:97. doi: 10.3389/fcimb.2013. 00097

Chao, G., Jiao, X., Zhou, X., Wang, F., Yang, Z., Huang, J., et al. (2010). Distribution of genes encoding four pathogenicity islands (VPaIs), T6SS, biofilm, and type I pilus in food and clinical strains of Vibrio parahaemolyticus in China. Foodborne Pathog. Dis. 7, 649-658. doi: 10.1089/fpd.2009.0441

Fujino, T., Okuno, Y., Nakada, D., Aoyama, A., Fukai, K., Mukai, T., et al. (1953). On the bacteriological examination of shirasu-food poisoning. Med. J. Osaka Univ. 4, 299-304.

Gonzalez-Escalona, N., Blackstone, G. M., and DePaola, A. (2006). Characterization of a Vibrio alginolyticus strain, isolated from Alaskan oysters, carrying a hemolysin gene similar to the thermostable direc themolysin-related hemolysin gene (trh) of Vibrio parahaemolyticus. Appl. Environ. Microbiol. 72, 7925-7929. doi: 10.1128/AEM.01548-06

Gotoh, K., Kodama, T., Hiyoshi, H., Izutsu, K., Park, K. S., Dryselius, R., et al. (2010). Bile acid-induced virulence gene expression of Vibrio parahaemolyticus reveals a novel therapeutic potential for bile acid sequestrants. PLOS ONE 5:e13365. doi: 10.1371/journal.pone.0013365

Hara-Kudo, Y., Saito, S., Ohtsuka, K., Yamasaki, S., Yahiro, S., Nishio, T., et al. (2012). Characteristics of a sharp decrease in Vibrio parahaemolyticus infections and seafood contamination in Japan. Int. J. Food Microbiol. 157, 95-101. doi: 10.1016/j.ijfoodmicro.2012.04.019

Hiyoshi, H., Kodama, T., Iida, T., and Honda, T. (2010). Contribution of Vibrio parahaemolyticus virulence factors to cytotoxicity, enterotoxicity, and lethality in mice. Infect. Immun. 78, 1772-1780. doi: 10.1128/IAI.01051-09

Honda, T., Ni, Y. X., and Miwatani, T. (1988). Purification and characterization of a hemolysin produced by a clinical isolate of Kanagawa phenomenon-negative Vibrio parahaemolyticus and related to the thermostable direct hemolysin. Infect. Immun. 56, 961-965.

Honda, T., Ni, Y., Miwatani, T., Adachi, T., and Kim, J. (1992). The thermostable direct hemolysin of Vibrio parahaemolyticus is a pore-forming toxin. Can. J. Microbiol. 38, 1175-1180. doi: 10.1139/m92-192

Izutsu, K., Kurokawa, K., Tashiro, K., Kuhara, S., Hayashi, T., Honda, T., et al. (2008). Comparative genomic analysis using microarray demonstrates a strong correlation between the presence of the 80 -kilo base pathogenicity island and pathogenicity in Kanagawa phenomenon-positive Vibrio parahaemolyticus strains. Infect. Immun. 76, 1016-1023. doi: 10.1128/IAI. 01535-07

Jones, J. L., Ludeke, C. H. M., Bowers, J. C., Garrett, N., Fischer, M., Parsons, M. B., et al. (2012). Biochemical, serological, and virulence characterization of clinical and oyster Vibrio parahaemolyticus isolates. J. Clin. Microbiol. 50, 2343-2352. doi: 10.1128/JCM.00196-12

Kanungo, S., Sur, D., Ali, M., You, Y. A., Pal, D., Manna, B., et al. (2012). Clinical, epidemiological, and spatial characteristics of Vibrio parahaemolyticus diarrhea and cholera in the urban slums of Kolkata, India. BMC Pub. Health 12:830. doi: 10.1186/1471-2458-12-830

Kaysner, C. A., Abeyta, C. Jr., Stott, R. F., Lilja, J. L., and Wekell, M. M. (1990). Incidence of urea-hydrolyzing Vibrio parahaemolyticus in WillapaBay, Washington. Appl. Environ. Microbiol. 56, 904-907.

Kishishita, M., Matsuoka, N., Kumagai, K., Yamasaki, S., Takeda, Y., and Nishibuchi, M. (1992). Sequence variation in the thermostable direct hemolysin-related hemolysin (trh) gene of Vibrio parahaemolyticus. Appl. Environ. Microbiol. 58, 2449-2457.

Klein, S. L., Gutierrez West, C. K., Mejia, D. M., and Lovell, C. R. (2014). Genes similar to the Vibrio parahaemolyticus virulence-related genes tdh, th and vscC2 occur in other vibrionaceae species isolated from a pristine estuary. Appl. Environ. Microbiol. 80, 595-602. doi: 10.1128/AEM.02895-13

Li, Y., Xie, X., Shi, X., Lin, Y., Qiu, Y., Mou, J., et al. (2014). Vibrio parahaemolyticus, Southern Coastal Region of China, 2007-2012. Emerg. Infect. Dis. 20, 685-688. doi: 10.3201/eid2004.130744 
Lynch, T., Livingstone, S., Buenaventura, E., Lutter, E., Fedwick, J., Buret, A. G., et al. (2005). Vibrio parahaemolyticus disruption of epithelial cell tight junctions occurs independently of toxin production. Infect. Immun. 73, 1275-1283. doi: 10.1128/IAI.73.3.1275-1283.2005

Mahoney, J. C., Gerding, M. J., Jones, S. H., and Whistler, C. A. (2010). Comparison of the pathogenic potentials of environmental and clinical Vibrio parahaemolyticus strains indicates a role for temperature regulation in virulence. Appl. Environ. Microbiol. 76, 7459-7465. doi: 10.1128/AEM.01450-10

Matsuda, S., Kodama, T., Okada, N., Okayama, K., Honda, T., and Iida, T. (2010). Association of Vibrio parahaemolyticus thermostable direct hemolysin with lipid rafts is essential for cytotoxicity but not hemolytic activity. Infect. Immun. 78, 603-610. doi: 10.1128/IAI.00946-09

Matsumoto, C., Okuda, J., Ishibashi, M., Iwanaga, M., Garg, P., Ramamurthy, T., et al. (2000). Pandemic spread of an O3:K6 clone of Vibrio parahaemolyticus and emergence of related strains evidenced by Arbitrarily Primed PCR and toxRS sequence analysis. J. Clin. Microbiol. 38, 578-585.

Matz, C., Nouri, B., McCarter, L., and Martinez-Urtaza, J. (2011). Acquired type III secretion system determines environmental fitness of epidemic Vibrio parahaemolyticus in the interaction with bacterivorous protists. PLoS ONE 6:e20275 doi: 10.1371/journal.pone.0020275

Ming, X., Yamamoto, K., and Honda, T. (1994). Construction and characterization of an isogenic mutant of Vibrio parahaemolyticus having a deletion in the thermostable direct hemolysin-related hemolysin gene (trh). J. Bacteriol. 176, $4757-4760$

Nelapati, S., Nelapati, K., and Chinnam, B. K. (2012). Vibrio parahaemolyti cus-An emerging foodborne pathogen-A Review. Vet. World 5, 48-62. doi: 10.5455/vetworld.2012.48-63

Nishibuchi, M., and Kaper, J. B. (1995). Thermostable direct hemolysin gene of Vibrio parahaemolyticus: avirulence gene acquired by a marine bacterium. Infect. Immun. 63, 2093-2099.

Noriea, N. F. III, Johnson, C. N., Griffitt, K. J., and Grimes, D. J. (2010). Distribution of type III secretion systems in Vibrio parahaemolyticus from the northern Gulf of Mexico. J. Appl. Microbiol. 109, 953-962. doi: 10.1111/j.1365-2672.2010. 04722.x

Pace, J., and Chai, T. (1989). Comparison of Vibrio parahaemolyticus grown in estuarine water and rich medium. Appl. Environ. Microbiol. 55, 1877-1887.

Paranjpye, R., Hamel, O. S., Stojanovski, A., and Liermann, M. (2012). Genetic diversity of clinical and environmental Vibrio parahaemolyticus strains from the Pacific Northwest. Appl. Environ. Microbiol. 78, 8631-8638. doi: 10.1128/AEM. 01531-12

Park, K., Ono, T., Rokuda, M., Jang, M., Iida, T., and Honda, T. (2004a). Cytotoxicity and enterotoxicity of the thermostable direct hemolysin-deletion mutants of Vibrio parahaemolyticus. Microbiol. Immunol. 48, 313-318. doi: 10.1111/j.1348-0421.2004.tb03512.x

Park, K.-S., Ono, T., Rokuda, M., Jang, M.-H., Okada, K., Iida, T., et al. (2004b). Functional characterization of two type III secretion systems of Vibrio parahaemolyticus. Infect. Immun. 72, 6659-6665. doi: 10.1128/IAI.72.11.66596665.2004

Pazhani, G. P., Bhowmik, S. K., Ghosh, S., Guin, S., Dutta, S., Rajendran, K., et al. (2014). Trends in the epidemiology of pandemic and non-pandemic strains of Vibrio parahaemolyticus isolated from diarrheal patients in Kolkata, India. PLoS Negl. Trop. Dis. 8:2815. doi: 10.1371/journal.pntd.0002815
Raghunath, P., Karunasagar, I., and Karunasagar, I. (2009). Improved isolation and detection of pathogenic Vibrio parahaemolyticus from seafood using a new enrichment broth. Int. J. Food Microbio1. 129, 200-203. doi: 10.1016/j.ijfoodmicro.2008.11.026

Raghunath, P., Maiti, B., Shekar, M., Karunasagar, I., and Karunasagar, I. (2010). Clinical isolates of Aeromonas veronii biovar veronii harbor a non functional gene similar to the thermostable direct hemolysin-related hemolysin (trh) gene of Vibrio parahaemolyticus. FEMS Microbiol. Lett. 307, 151-157. doi: 10.1111/j.15746968.2010.01974.x

Salomon, D., Gonzalez, H., Updegraff, B. L., and Orth, K. (2013). Vibrio parahaemolyticus type VI secretion system 1 is activated in marine conditions to target bacteria, and is differentially regulated from system 2. PLOS ONE 8:61086. doi: 10.1371/journal.pone.0061086

Shimohata, T., and Takahashi, A. (2010). Diarrhea induced by infection of Vibrio parahaemolyticus. J. Med. Invest. 57, 179 -182. doi: 10.2152/jmi.57.179

Shinoda, S. (2011). Sixty years from the discovery of Vibrio parahaemolyticus and some recollections. Biocontrol Sci. 16, 129-137. doi: 10.4265/bio.16.129

Su, C. Y., and Liu, C. (2007). Vibrio parahaemolyticus: a concern of seafood safety. Res. Microbiol. Elsevier. 24, 549-558. doi: 10.1016/j.fm.2007.01.005

Takahashi, A., Kenjyo, N., Imura, K., Myonsun, Y., and Honda, T. (2000). Cl- secretion in colonic epithelial cells induced by the Vibrio parahaemolyticus hemolytic toxin related to thermostable direct hemolysin. Infect. Immun. 68, 5435-5438. doi: 10.1128/IAI.68.9.5435-5438.2000

Yu, W. T., Jong, K. J., Lin, Y. R., Tsai, S. E., Tey, Y. H., and Wong, H. C. (2013). Prevalence of Vibrio parahaemolyticus in oyster and clam culturing environments in Taiwan. Int. J. Food Microbiol. 160, 185-192. doi: 10.1016/j.ijfoodmicro.2012.11.002

Yu, Y., Yang, H., Li, J., Zhang, P., Wu, B., Zhu, B., et al. (2012). Putative type VI secretion systems of Vibrio parahaemolyticus contribute to adhesion to cultured cell monolayers. Arch. Microbiol. 194, 827-835. doi: 10.1007/s00203-012-0816-Z

Zhang, L., and Orth, K. (2013). Virulence determinants for Vibrio parahaemolyticus infection. Curr. Opin. Microbiol. 16, 70-77. doi: 10.1016/j.mib.2013.02.002

Zhang, X. H., and Austin, B. (2005). Haemolysins in Vibrio species. J. Appl. Microbiol. 98, 1011-1019. doi: 10.1111/j.1365-2672.2005.02583.x

Conflict of Interest Statement: The author declares that the research was conducted in the absence of any commercial or financial relationships that could be construed as a potential conflict of interest.

Received: 01 December 2014; accepted: 30 December 2014; published online: 22 January 2015.

Citation: Raghunath P (2015) Roles of thermostable direct hemolysin (TDH) and TDH-related hemolysin (TRH) in Vibrio parahaemolyticus. Front. Microbiol. 5:805. doi: $10.3389 /$ fmicb.2014.00805

This article was submitted to Food Microbiology, a section of the journal Frontiers in Microbiology.

Copyright (c) 2015 Raghunath. This is an open-access article distributed under the terms of the Creative Commons Attribution License (CC BY). The use, distribution or reproduction in other forums is permitted, provided the original author(s) or licensor are credited and that the original publication in this journal is cited, in accordance with accepted academic practice. No use, distribution or reproduction is permitted which does not comply with these terms. 